\title{
Medical Physics and Imaging-A Timely Perspective
}

\author{
Thomas Beyer $^{1 *}$, Dale L. Bailey ${ }^{2}$, Udo J. Birk ${ }^{3}$, Irene Buvat ${ }^{4}$, Ciprian Catana ${ }^{5}$, \\ Zhen Cheng ${ }^{6}$, Qiyin Fang ${ }^{7}$, Federico Giove ${ }^{8}$, Claudia Kuntner ${ }^{9}$, Elmar Laistler ${ }^{10}$, \\ Francesco Moscato ${ }^{11}$, Stephan G. Nekolla ${ }^{12}$, Ivo Rausch ${ }^{1}$, Itamar Ronen ${ }^{13}$, \\ Simo Saarakkala ${ }^{14}$, Kris Thielemans ${ }^{15}$, Wouter van Elmpt ${ }^{16}$ and Ewald Moser ${ }^{10}$
}

${ }^{1}$ Quantitative Imaging and Medical Physics (QIMP) Team, Center for Medical Physics and Biomedical Engineering, Medical University of Vienna, Vienna, Austria, ${ }^{2}$ Sydney Medical School, University of Sydney, Sydney, NSW, Australia, ${ }^{3}$ University of Applied Sciences of the Grisons Chur, Chur, Switzerland, ${ }^{4}$ Centre National de la Recherche Scientifique (CNRS), Paris, France, ${ }^{5}$ Athinoula A. Martinos Center for Biomedical Imaging, Department of Radiology, Massachusetts General Hospital and Harvard Medical School, Boston, MA, United States, ${ }^{6}$ Stanford University, Stanford, CA, United States, ${ }^{7}$ McMaster University, Hamilton, ON, Canada, ${ }^{8}$ Centro Ricerche Enrico Fermi, and Fondazione Santa Lucia, Rome, Italy, ${ }^{9}$ Preclinical Molecular Imaging, AlT Austrian Institute of Technology GmbH, Seibersdorf, Austria, ${ }^{10} \mathrm{MR}$ Centre of Excellence, Center for Medical Physics and Biomedical Engineering, Medical University of Vienna, Vienna, Austria, ${ }^{11}$ Center for Medical Physics and Biomedical Engineering, Medical University of Vienna, Vienna, Austria, ${ }^{12}$ Faculty of Medicine, Technical University of Munich, Munich, Germany, ${ }^{13}$ Leiden University Medical Center, Leiden, Netherlands, ${ }^{14}$ Research Unit of Medical Imaging, Physics and Technology, University of Oulu, Oulu, Finland, ${ }^{15}$ University College London, London, United Kingdom, ${ }^{16}$ Department of Radiation Oncology (MAASTRO), GROW - School for Oncology, Maastricht University Medical Centre+, Maastricht, Netherlands

Keywords: medical physics and biomedical engineering, medical imaging, image data, artificial intelligence, imaging technology

\section{OPEN ACCESS}

Edited and reviewed by: Alex Hansen,

Norwegian University of Science and Technology, Norway

*Correspondence: Thomas Beyer thomas.beyer@meduniwien.ac.at

Specialty section:

This article was submitted to Medical Physics and Imaging,

a section of the journal Frontiers in Physics

Received: 28 November 2020 Accepted: 06 April 2021 Published: 12 May 2021

Citation:

Beyer T, Bailey DL, Birk UJ, Buvat I, Catana C, Cheng Z, Fang Q, Giove F, Kuntner C, Laistler E, Moscato F Nekolla SG, Rausch I, Ronen I, Saarakkala S, Thielemans $K$, van Elmpt W and Moser E (2021) Medical Physics and Imaging-A Timely

Perspective. Front. Phys. 9:634693. doi: 10.3389/fphy.2021.634693
The well-being of people is an anchor point for humanities. Over the past millennia generations of medical professionals have risen from early representatives of small groups of people with a gift for titrating herbal medicine for wound healing and handling fever to widely diversified medical experts with a wide access to complex technologies that extend far beyond a timber-cast for stabilizing broken joints. Today, medicine, through its numerous disciplines and disciples who engage in all stages of patient management, from disease prediction, through screening, diagnosing, phenotyping, therapeutic interventions, follow-up and post-intervention or palliative care has diversified in a way that it can no longer be represented by a single medicinal man as it was fathomable few 100 years ago. Instead, medicine has become a multi-disciplinary, complex and integral domain of our societal ecosystems. In today's healthcare systems, medical professionals employ technologies and instruments that frequently have been proposed, devised and manufactured by non-medical professionals. To that extent, medical physicists, in particular, play a key role in supporting medical professionals and, subsequently, in helping patients. Physicists, or natural philosophers, as they have been called before, have supported medical diagnosis and treatment for over 4,000 years [1].

Over the decades we witnessed the pace of evolution in medicine, together with its technical and methodological complements. Next to medical physics, these complementary disciplines include social sciences, ethics, chemistry, and biology, to name a few. All of them engage people and teams who seek to contribute visibly and responsibly to societal healthcare endeavors. In that regard, our journal "Frontiers in Physics-Medical Physics and Imaging" provides a valuable platform specifically to physicists and imaging scientists of all traits but with an appreciation for the role of applied medical physics, and medical imaging in particular.

Our journal was launched in 2013 as a subspecialty for Biomedical Physics covering various imaging techniques in experimental and human research, radiation physics and technology and robust statistics [2]. In 2018, the journal name was changed to "Medical Physics and Imaging" followed by a handover of the editor-in-chief position. Today, our subspecialty journal has 
published $>200$ articles and about 20 Research Topics 255 articles hits/downloads. In 2019, 6 years into the launch of the journal, we did receive our first impact factor (1.895), which is a tribute to the tireless efforts of the editors, reviewers, and above all, the authors who chose this platform to have their work peer-reviewed and published. Only 1 year later in 2020, the impact factor has risen to 2.67 , which is a testimony to the increasing value of our journal and its outreach into the relevant communities.

There are numerous journals that cater to the scientists in the field of applied physics, and the numbers are changing continuously as this field is growing with more and more manuscripts being published. The authors consent to the observation that with its inception this journal has made a serious effort to cater to the medical applications of physics, to render physics-driven innovation palatable to scientists and clinical adopters. In that regard, recently accepted manuscripts cover an exceptionally broad range of topics, including magnetic resonance imaging (MRI) with low- and high magnetic field strengths [3-5], LASER scanning microscopy techniques [6], applications of dual-channel endoscopy [7], small-animal SPECT [8] and total-body PET imaging [9], and preclinical ultrasound systems [10]. Likewise, relevant publications in our journal address conceptual measurements of basic biological and morphological parameters, such as carotid stiffness [11], cardiac arrhythmia [12], articular cartilage constituents [13] as well as the ultrastructure of bone [14, 15]. Moreover, key publications address concepts of therapeutic applications, such as a transcranial MRI-guided focused US treatment [16] and cancer immunotherapy [17].

This breadth of medical physics being applied in medicine is further illustrated by the 25 Research Topics that were launched by our journal since its inception; such as on "Status Go for Preclinical Imaging", "Multi-modality Molecular Imaging" [https://www.frontiersin.org/research-topics/5947/multimodalitymolecular-imaging] and "Innovative Developments in MultiModality Elastography" [https://www.frontiersin.org/researchtopics/13038/innovative-developments-in-multi-modality-

elastography.]

Consider the word "imaging" in the name of our journal. Imaging is a means, provided through technological and methodological advances, to view the human body from outside and inside, to probe anatomical, functional and even molecular and signaling pathways non-invasively for new information of the state of the patient for rendering an accurate and personal diagnosis. Medical imaging, by means of radiology and molecular imaging, has become a foundation for diagnosis and therapy planning in numerous diseases that patients present with [18]. What is more, imaging is being used extensively in healthy subjects, or volunteers to better understand physiology and signaling, for example in the brain, so as to build reference models of normal physiology, biology and functional correlates that can be employed to better define abnormal variants during diagnostic and therapeutic work-up of patients. Hence, the growing field of medical, clinical, or diagnostic imaging (MCDI) represents a focus of our journal (Figure 1), and related manuscripts help our readers appreciate the corner blocks for high-quality diagnosis based on multi-parameter and multimodality imaging techniques.

Of interest, the seven most frequently used medical imaging techniques nowadays are X-ray (i.e., conventional radiography), CT, PET, SPECT, OI, US, and MRI. The first two (X-ray and CT) make use of high-energy photons to produce 2D- or 3Dimage sets of the anatomy of living organisms. In contrast, nuclear medicine techniques, such as PET and SPECT employ small amounts of radioactively labeled tracers that participate in the metabolic and signaling pathways in the organism, whereby their distribution and quantities can be measured through the emitted radiation. Finally, MRI, OI, and US employ non-ionizing radiation for diagnostic purposes, i.e., mechanical waves in the $\mathrm{MHz}$ range (US), light (OI), and magnetic fields, also resonating in the $\mathrm{MHz}$ range (MRI), respectively. In general, most of the imaging techniques listed above, but also therapeutic approaches, use electromagnetic radiation in a wide range of frequencies or energies (Figure 2A). These parameters partly determine penetration depth, spatial resolution, specific absorption rate, etc. In turn, this affects sensitivity and specificity of the medical imaging techniques applied (Figure 2B).

In the following, we shall highlight key areas of entanglement of medical and imaging physicists with clinical research and healthcare, particularly in view of the innovating imaging aspects during patient diagnosis and treatment planning and follow-up.

\section{X-RAY AND CT}

Transmission imaging based on the use of X-rays helps generate planar images that-upon repeated scanning-can be used to sample dynamic processes (fluoroscopy), as well as 3D- and 4Dcomputerized tomography (CT) data. Transmitted signals from a narrow X-ray beam traversing the subject are used for image reconstruction to form planar (X-ray) and cross-sectional (CT) images of the subject under investigation (Figure 3). CT is a tomographic imaging method with systems that comprise of 100 , or more detector rows covering an axial field-of-view of several $\mathrm{cm}$. CT generates contiguous axial images of sub- $\mathrm{mm}$ resolution, which can be digitally stacked to form 3-dimensional, high-resolution images of the investigated area; the preferred acquisition mode today is the spiral scan mode [19].

CT images are highly quantitative and reproducible and can be provided with very good contrast resolution. While being primarily an anatomical imaging modality, $\mathrm{CT}$ has been shown to also yield functional data through dynamic perfusion scanning. These perfusion protocols perform a $4 \mathrm{D}$ acquisition by repeatedly imaging the same body region in time steps of 3 to $5 \mathrm{~s}$ for about $30 \mathrm{~s}$ post administration of the contrast agent. Using the socalled "Time attenuation curve (TAC)" of a voxel, important hemodynamic parameters, such as the blood flow and volume can be derived [20].

Many of the technological and methodological developments in X-ray and CT imaging arise from the concerns over radiationinduced biological risks due to singular, high-dose and multiple transmission scans using ionizing radiation. These concerns have led to a wide number of efforts to reduce the dose in CT, 


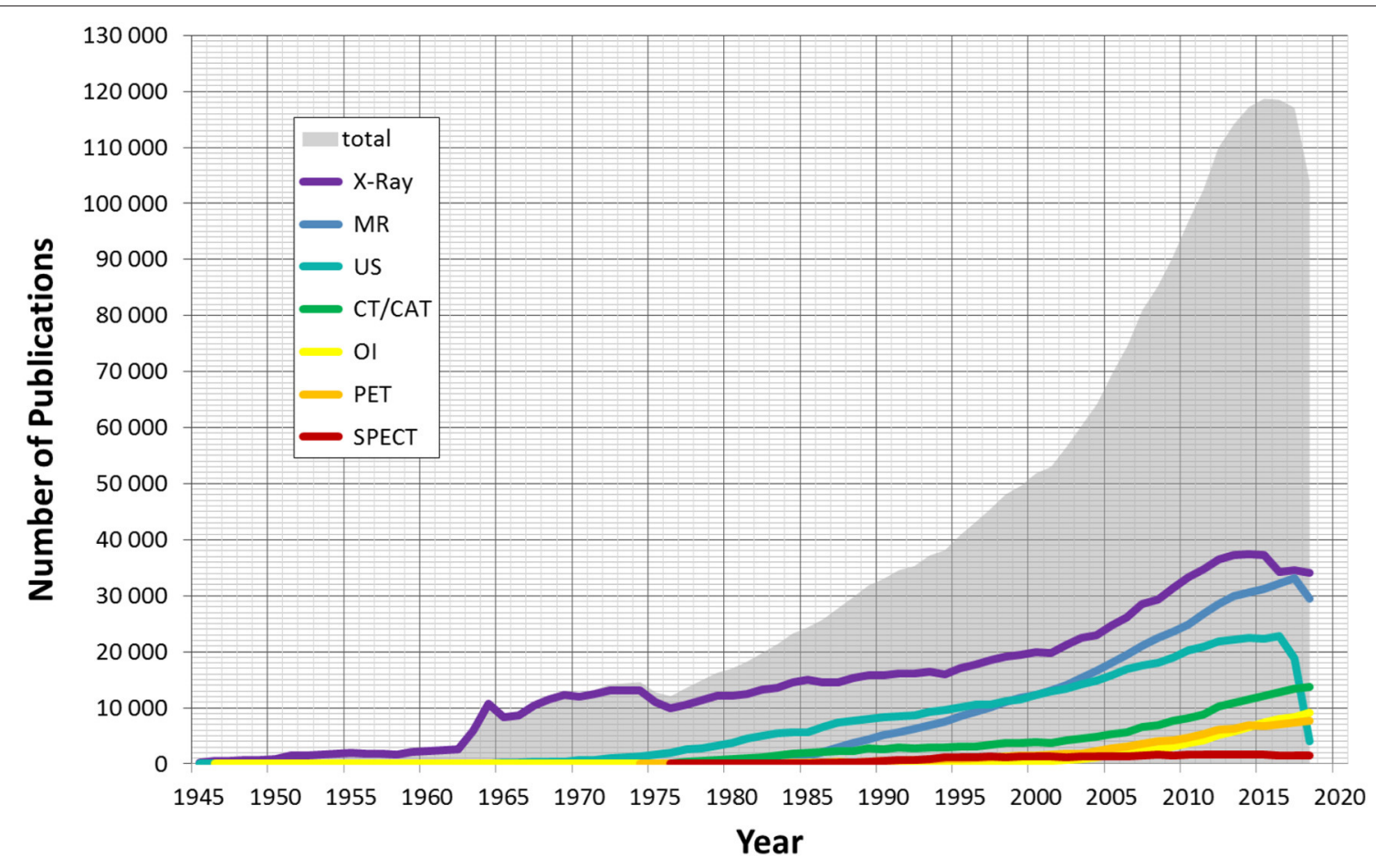

FIGURE 1 | MCDI papers listed in PubMed (gray, shaded area) between 1945 and 2018 (search April 2020: (medical) OR (clinical) OR (diagnostic) AND imaging). Note, imaging papers published annually increased by a factor of 1,000 from 1945 to 2018. The colored lines represent papers published on one of the seven most popular imaging methods: X-ray (Purple) and CT (Green), SPECT (Red) and PET (Orange), US (Light blue) and MRI (Dark blue), and OI (Yellow).

and include the use of automated modulation of tube currents, exposure, or tube voltage selection. More recently, hardware design changes that include powerful X-ray tubes that allow for thicker prefiltration, highly-integrated detectors with less internal noise, as well as iterative image reconstruction techniques [21] have been proposed. These techniques make more efficient use of the X-ray dose and help reduce the X-ray dose while preserving image quality.

X-ray transmission imaging has been an active area of engagement for clinical and research physicists. Together with engineers and medical users, a number of innovations have been developed and validated that help improve the efficacy of X-ray imaging (i.e., better image quality at lower exposure levels to patients), or helped deduce novel biomarker information. These developments include, but are not limited to: dual-energy CT (DECT) to exploit differences in the energy dependency of the attenuation coefficients and help selectively display materials that might appear at the same CT value in single-energy CT (Dual Energy CT in Oncology. 2015, ISBN 9783319195629) and photon counting through the use of novel X-ray detectors that convert the $\mathrm{X}$-ray photon directly into an electric signal that is descriptive of the photon energy [22].

\section{NUCLEAR MEDICINE PHYSICS}

Nuclear Medicine is based on the use of radioactive isotopes as probes to track physiological processes ("tracer principle") or to deliver therapeutic doses to specific targets ("internal radiation therapy"). The measurement of the emitted radiation (Figure 3) arising from the decay of the isotopes, it for imaging or dosimetry, is one of the fundamental skills of an imaging physicist. Physicists have been, and are, integral in the conception, development and improvement of detection systems, the development and implementation of correction methods for various effects limiting quantitative and qualitative readings and in the development of reconstruction algorithms for tomographic applications [23].

Recent examples of these engagements in research and development of imaging systems include the introduction of solid-state based photo-detectors [24], the design and validation of a total body PET system [25], a novel PET system based on plastic scintillators (J-PET) and Compton cameras [26]. Further, progress was made, for example, with the introduction of MR-based attenuation correction for PET/MRI systems, Monte Carlo based scatter correction methods and advanced reconstruction algorithms for SPECT and PET. In addition to these rather technical engagements, medical physicists are involved in all aspects of clinical operation in research and routine, including isotope production using cyclotrons or generator systems, radiation protection issues, study and imaging protocol designs, operations and maintenance of the imaging systems, personalized dosimetry calculations and radioactive waste management. 

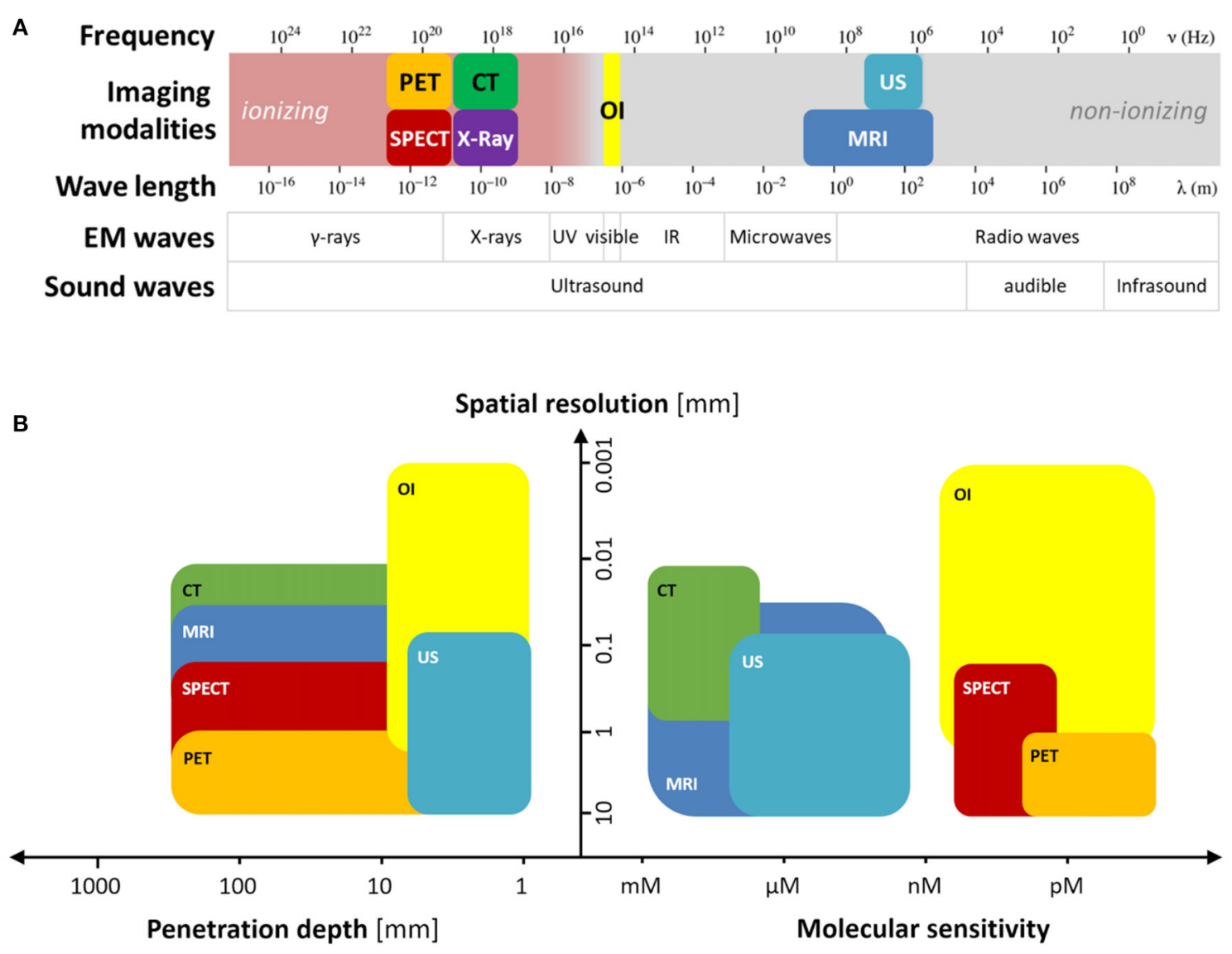

FIGURE 2 | (A) Electromagnetic frequency spectrum with indications for frequency ranges employed for various imaging modalities. (B) Key imaging performance illustrated for most clinical imaging modalities as a function of spatial resolution, penetration depth into tissue (left) and molecular sensitivity. The same color code as that in Figure $\mathbf{1}$ is used.

In view of the close interaction with clinical partners, the application of Nuclear Medicine physics faces two significant challenges in the future: (i) to seamlessly integrate the medical physicist into the multi-disciplinary clinical team managing the twenty-first century patient by understanding the disease, its pathological, genomic and biochemical basis, and the role that diagnostic imaging and radionuclide therapy can play in managing the patient, and, (ii) to expand our knowledge of the interaction of radiation with the body and the fundamental radiobiological changes it induces in order to make the most effective and safe use of the powerful tools that we now have at our disposal. This includes multi-parametric imaging as a prerequisite for changing the current use of radionuclide therapies from being based on empirically determined standard procedures and dosage to a patient specific treatment adaption, rendering a condition such as cancer to be a chronic, but manageable, illness that the patient dies with rather than from.

These challenges require the nuclear medicine physicist to speak the language of their clinical partners and to be able to offer insight into the likely outcome for a patient subjected to a nuclear medicine procedure [27]. Nuclear medicine physicists are usually those within the multi-disciplinary team who have the most complete understanding of the science involved in the measurements or imaging of the patients. Clinicians are typically highly skilled at recognizing abnormal patterns in disease, while a physicist is expected to understand the complete diagnostic or therapeutic workflow from the radioactive compound used through to the imaging or measurement instrumentation involved (and associated errors), any computer analysis and interpretation of results, and, thus, is expected to recognize any divergence from normality in any part of the chain.

\section{ULTRASOUND PHYSICS AND APPLICATIONS}

Ultrasound (US) is a sound wave with frequency higher than the average human audible level (e.g., $20 \mathrm{kHz}-100 \mathrm{MHz}$ ). Reflection of ultrasound is the result of mechanical property differences at the interface of different structures (Figure 3). Measuring these reflections allows ultrasound to non-invasively probe structures inside of an object, such as the human body. Since modern electronics and transducer technology can relatively easily handle ultrasound frequencies, ultrasound imaging quickly 

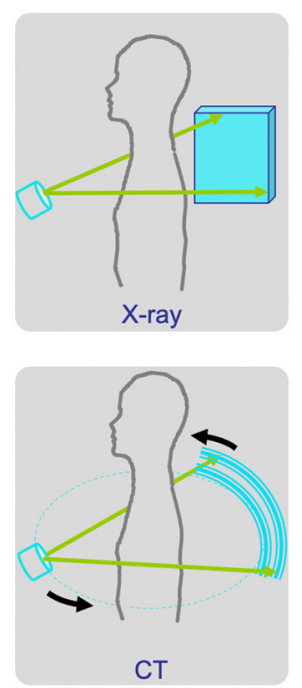

Structural imaging
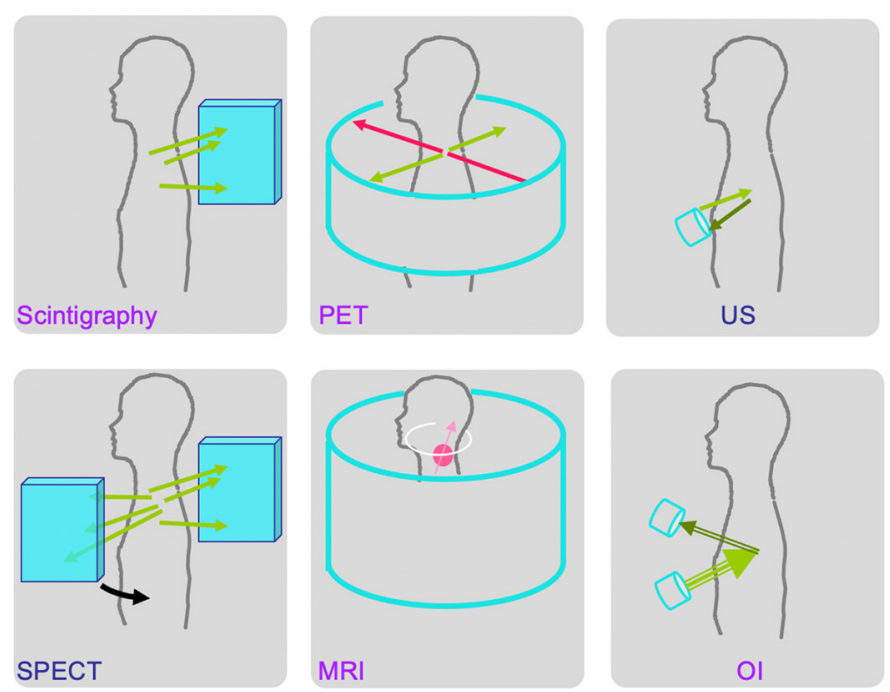

Functional imaging

FIGURE 3 | Basic principles of key imaging modalities that fall into the categories of structural (anatomical) and functional (metabolic) imaging as indicated in blue and purple, respectively: X-ray and computed tomography (CT) transmission imaging, nuclear medicine scintigraphy, positron emission tomography (PET) and single photon emission computed tomography (SPECT), ultrasound (US), optical imaging (OI), and magnetic resonance imaging (MRI).

became a standard clinical imaging modality and has been widely used in many medical diagnostic procedures. Compared with other medical imaging modalities, such as CT and MRI, ultrasound is a low-cost modality and, most importantly, allows real time imaging at bedside. Conventional ultrasound imaging techniques, however, have lower specificity and lower spatial resolutions compared with CT and MRI.

In the past 10 years, Ultrasound technologies have seen many new advances. Most notably, they benefited from the introduction of new materials and designs in transducer technologies and high-speed digitizers, high spatial resolution, and microscopy-like imaging capabilities that can be achieved through high-frequency ultrasound [28]. Functional and specific imaging can also be achieved by using new contrast enhancing agents, such as microbubbles [29]. Another important development in ultrasound's medical application is the therapeutic use of high-intensity focused ultrasound (HIFU), which also uses the microbubbles [30]. HIFU allows selective ablation of targets inside the body with little collateral damages to the surrounding tissue. Another promising area is the use of ultrasound in targeted drug delivery applications [31].

As we are entering a new decade, there are many potential research areas worth exploring in medical ultrasound. First, ultrasonic systems are becoming more compact and flexible, to an extent that wearable ultrasound devices come within reach [32, 33]. Such technology would allow, for example, the monitoring of mobility and physiological parameters. Integration of ultrasound with other diagnostic and therapeutic modalities (e.g., CT, MRI) is also an active area in the domain of medical physics and imaging [16]. New algorithms using artificial intelligence and machine learning for real time $3 \mathrm{D}$ reconstruction and machine assisted diagnosis are other emerging areas of significant relevance [34].

\section{MRI PHYSICS AND APPLICATIONS}

Magnetic resonance imaging (MRI) is a medical imaging technique that uses magnetic fields and radio waves to create non-invasive images from functional/molecular information of organs and tissues (Figure 3). The very low energy associated to Zeeman splitting induced by a (strong) static magnetic field is tightly related to many features that make MRI an invaluable technique for the clinic, which include the coherence of the emitted radiation as the basis for Fourier-based imaging with position encoded by field gradients [35]. The very same low energy is also the root cause of the limited sensitivity of the technique. Higher magnetic fields result in stronger nuclear polarization [3] and, subsequently, in an increase in induced currents in the coils that translates into a quadratic increase in detected signal. An increase in signal not only allows for faster or better resolved scans, but also ushers imaging and spectroscopic measurements of low-sensitivity nuclei to clinical scanners [36].

Advances in stronger and faster switching gradient coils, applied at ultra-high magnetic fields, may open a new and potentially game changing route in high-resolution MRI [37]. The clinical value of imaging and spectroscopy of nuclei, such as ${ }^{23} \mathrm{Na},{ }^{31} \mathrm{P},{ }^{2} \mathrm{D}$, and ${ }^{13} \mathrm{C}$ is currently being investigated. In line with improvements of MRI signals, there is a proliferation of methods for in-plane or between-slices scan acceleration that is increasingly shaping the technology behind high field (1.5-3 T), or ultra-high field (seven Tesla and beyond) MRI. Undeniably, 
increased complexity, costs and sheer bulk of ultra-high MRI systems play a fundamental role in the resistance to integration of these systems in clinical practice.

One of the key challenges that MRI physicists are faced with is how to back-port recent developments in encoding and readout technique to low field systems. There are some advantages for MRI at low field that can be leveraged to increase image quality and contrast to offset the predicament of signal loss: longer $\mathrm{T}_{2}^{*}$, shorter $\mathrm{T}_{1}$ and lower SAR allow the use of RF-heavy techniques that suffer from severe limitations at higher field. In addition, the risks to patients caused by the high static and radiofrequency magnetic fields are minimized, making the exclusion criteria for patient scanning much less restrictive.

Lately, MRI physicists have become engaged with the introduction of quantitative MRI methods to assist clinical decision making and patient management. For example, in clinical neuroimaging, the most widely used MRI modalities are the qualitative $\mathrm{T}_{1}$-weighted, $\mathrm{T}_{2}$-weighted (mostly in conjunction with suppression of the cerebrospinal fluid contribution with FLAIR) and diffusion weighted images. The crispness and highly detailed character of these qualitative images make them more attractive to neuroradiologists than their quantitative counterparts $-\mathrm{T}_{1}$ and $\mathrm{T}_{2}$ maps.

Quantitative MRI has permeated the clinic with other modalities, such as cerebral blood flow mapping using arterial spin labeling (ASL) [38], microstructural imaging using diffusion tensor imaging (DTI) metrics [39] and localized, high-resolution spectroscopic imaging [40]. Quantitative MRI holds the promise of decreasing variance in imaging metrics by isolating the effect of one specific contrast mechanism, but are not immune to other problems such as error propagation and inter-vendor differences in scanning sequences.

\section{OPTICAL IMAGING}

Optical imaging (OI) uses light, often from LASERs and LEDs, and enables imaging with good contrast at high spatial resolution, albeit with strongly limited penetration depth into tissue (Figure 3). For these reasons, many applications of optical imaging are targeting cultured cells or fixed cell samples; for instance, optical microscopy is one of the methods of choice not only in histopathology, but also for studying cell development and cell fate, for genetic expression analyses, for the analysis of cell-pathogen interactions, cellular and subcellular signaling, metabolism and cell-cell interactions.

Routine medical application of optical imaging target surface and transparent parts of the human body (e.g., in dermatology, ophthalmology, various endoscopic procedures, and dentistry). Nonetheless, a number of additional optical imaging methods were devised for imaging normal and diseased patients and animals in clinical and pre-clinical settings [41].

OI is used mainly to image the human skin, the eye, and other accessible body parts [42], such as teeth, mucus, pharynx, colon, etc. For this purpose, multi-photon imaging [9] and optical coherence tomography (OCT) [43, 44] are the OI techniques most frequently employed. Further, the use of highly-specific markers, such as fluorescent tags or novel imaging probes, promotes the adoption of OI for in-vivo imaging [45]. Currently available 3D optical imaging approaches include 2Photon microscopy, OCT, Light-Field Microscopy [46], Diffuse Optical Tomography [47], Optical Projection Tomography [48], Light-Sheet Microscopy [49] as well as Photoacoustics [50], an approach in which LASER light is used for illumination and contrast in combination with ultrasonic detection. More recently, a super-resolution microscopy variant of OI was proposed that permits non-invasive investigations with spatial resolutions below $10 \mathrm{~nm}[51,52]$.

To further improve the in vivo imaging performance in living subject, imaging at the second near-infrared wavelength window (NIR-II: $1,000-1,700 \mathrm{~nm}$ ) has been heavily pursued as a novel and attractive strategy [53]. NIR-II imaging has shown many advantages, such as deep tissue penetration, high resolution and imaging contrast, low autofluorescence, photon scattering and absorption. As such, NIR-II fluorescent endoscopy appears as a promising approach for intraoperative imaging $[54,55]$.

\section{COMBINED, OR HYBRID IMAGING AND RELATED PHYSICS}

Hybrid imaging denotes hardware combinations of complementary, dual-modality imaging methods, such as PET/CT, SPECT/CT and PET/MRI, which continued to evolve over the last decade and are now being all available for clinical use. PET/CT is the most mature and widely adopted hybrid imaging modality. The introduction of solid-state photon detectors (i.e., silicon photomultipliers) coupled with improved readout electronics have supported the adoption of the time-offlight (TOF) acquisitions in PET imaging [56]. Consequently, state-of-the-art TOF PET systems can be used to reconstruct images with higher signal-to-noise ratio, and to help speed up the data acquisition or lower the injected dose. Image quality was also improved by using digital photon counters and a one-to-one coupling of the individual scintillator crystals to the detector elements [57].

Very recently, PET systems with extra-long axial fields of view of almost $2 \mathrm{~m}$ were introduced, allowing the total body to be imaged within a single bed position and without moving the patient during the exam. First performance characterization studies demonstrated a more than two orders of magnitude sensitivity gain and several potential applications are currently being explored $[58,59]$.

SPECT/CT has also continued to mature and current systems combine latest generation collimator designs, solid-state detectors (i.e. cadmium-zinc-telluride) for SPECT with high performance diagnostic CT scanners. Statistical iterative image reconstruction algorithms have been implemented for SPECT and several vendors offer CT-based attenuation, scatter and even motion correction techniques for improved quantification [18]. Of note, one vendor provides a tri-modality SPECT-CT-PET system for maximum versatility [60].

In 2020, fully-integrated PET/MRI systems celebrate their 10th anniversary. Although three vendors are now 
commercializing fully-integrated PET/MRI for human imaging, the adoption of this technology has been slower than that of PET/CT, with only about 250 systems currently being installed around the world. Nevertheless, PET/MRI is used more and more broadly for applications in oncology, neurology and cardiology. Numerous groups have developed MR-based motion correction approaches for brain and whole-body application and several such techniques are already implemented on the commercially available scanners for routine use.

Medical imaging physicists have played a key role in the development, optimization and clinical adoption of all these hardware and software advances. As hybrid imaging modalities are inherently multimodal, it is essential for physicists with different areas of expertise to collaborate closely. This is perhaps the most obvious in the case of PET/MRI, where MRI physicists are needed for setting up the MR protocols for dedicated and whole-body data acquisition, while the PET physicists must handle the PET data processing (including MR-based attenuation correction) and image reconstruction aspects [61].

Looking further into the future, multi-modality imaging will need to be integrated with multi-omics (genomics, proteomics, metabolomics, transcriptomics, etc.) data. Building on the success of radiogenomics in combining diverse imaging and genomics data for several applications, advanced machine learning algorithms have been suggested to further bridge the gap between imaging and multi-omics to enable a more comprehensive assessment of diseases [62]. Though this will require the future medical physicists to step even more outside of their comfort zone, it will also give them the unique opportunity to expand their role and become indispensable members of the larger community.

\section{PRECLINICAL IMAGING}

Over the past years, preclinical imaging has moved to an independent scientific field. Especially the transition from producing only colorful images to developing methodologies for image acquisition, image analysis and quantification has boosted the translation between basic and clinical research. In contrast to clinical imaging, medical physics input is not mandated by legislation; nonetheless, the close integration of medical physics expertise in preclinical molecular research has demonstrated increased efficiency and quality of the research. Since in preclinical imaging image acquisition parameters are less well-defined and are adjusted locally, expertise, such as provided through medical physics, as to the implications of changes in the acquisition and reconstruction parameters on image quality and accuracy is welcome and required to make the best use of the animal models.

In preclinical nuclear imaging some acceptance testing standards are available (e.g., NEMA Standards Publication NU 4-2008 Performance Measurements of Small Animal Positron Emission Tomographs) but do not exist for all the different imaging modalities. Knowledge about the different modalities and published guidelines [63] aid in setting up a good QA/QC program for preclinical imaging.
The most important contribution of medical physics in preclinical imaging is that to image quantification. In preclinical nuclear medicine image data can be acquired together with blood sampling in animal models as to allow absolute quantification. The application of kinetic modeling and the deduction of image parameters expressed in parametric form, which are easily comprehended without compromising accuracy enables than a transition of methodology but also results from basic to clinical research.

Finally, with the advances in correlated multimodal imaging (CMI) [64], where information about the same specimen are acquired with two or more complementary modalities across scales, medical physics can contribute in providing tools for image fusion and registration. This is quite challenging as CMI applications range from imaging of cells and tissues to whole organisms to gain a complete picture of biological and biomechanical processes. In addition, as pointed out by the authors, CMI would benefit from standardization of protocols, data handling, and the development of optimized and advanced implementations, which are exactly the areas where medical physics can make a substantial contribution.

\section{RT PHYSICS}

Radiotherapy (RT) is the process of directing ionizing radiation (e.g., electrons, photons, or particles, such as protons) to damage and destroy the cancer cells in the human body. Most frequently high-energy photons (6-18 MV) are used to reach deep-seated tumors, while for more superficial tumors high-energy electrons up to $25 \mathrm{MeV}$ are employed. Alternative radiation treatments are based on the use of charged particles, such as protons, with a number of such proton therapy centers opening up throughout the world [65]. The physical properties of protons are well-suited for radiotherapy because the Bragg-peak can be exploited to deposit all energy of the proton beam while leaving no radiation dose distal to the peak.

Throughout the entire radiation treatment process medical imaging plays key roles: from diagnosis to quantifying and outlining the tumor as well as normal tissues and organs to spare, toward image-guidance for treatment prior to and during a typical course of treatment that may span over 3 to 7 weeks of daily treatment fractions. Adaptation during treatment to possible changes of the anatomy is of utmost importance for high-quality treatment plans and safe radiation dose delivery to the right location. Such changes can be picked up through onboard, cone-beam CT systems that are attached to the treatment devices $[66,67]$, or optical surface scanning for monitoring the patient position or breathing motion flow [68].

The recent combination of MRI scanners with treatment machines (MR-Linacs) allows to push the integration of imaging and treatment even further allowing simultaneous visualization and treatment of the target $[69,70]$. Advances in RT hardware are being supplanted by the recent adoption and implementation of AI technologies for many steps of the radiotherapy treatment workflow. For tumor outlining and 
organs-at-risk segmentation, AI technology based on deeplearning is at the same level as human expertise [71, 72]. Further, image reconstruction approaches and radiation treatment plan optimization algorithms also benefit from AI-driven solutions to allow for fast and accurate results [73-75]. Finally, the complexities of integrating the various hardware and software components of standard and advanced RT workflows can be addressed only through a multi-disciplinary team effort that supports active interactions between medical physicists, medical doctors and technologists, along with engineers, IT and datascience engineers [76-79].

\section{ADDITIVE MANUFACTURING AND 3D-PRINTING}

Additive manufacturing, also commonly known as 3D-printing, has been applied to medicine since the 1990's. The unique possibility to manufacture complex-shaped objects by a computer-controlled layer-by-layer deposition of different materials is fascinating and at the same time very powerful. $3 \mathrm{D}$ printing allows to literally materialize digital objects into physical parts. Originally, this manufacturing technique was mainly used by mechanical engineers to "rapid-prototype" parts that were then produced by more conventional methods, but now $3 \mathrm{D}$ printing has gone well-beyond this initial use. Thus, medical 3D printing can be thought of as an extension of medical imaging into the real world, thereby enhancing diagnostics and pre-operative planning by presenting anatomical structures in three dimensions and even allowing to physically manipulate them. Beyond these unique possibilities, $3 \mathrm{D}$ printing also allows to manufacture patient-specific implants or surgical tools for precision medicine and to engineer multicellular biological tissue constructs for regenerative medicine purposes.

Medical problems arising from skeletal defects of various tissues were the first to be addressed by $3 \mathrm{D}$ printing [80]. Medical problems in dentistry, and oral surgery in particular, are similar to those in skeletal applications because of the hard-tissue context in which $3 \mathrm{D}$ printing could be successfully applied [81]. While the original intent of $3 \mathrm{D}$ printing was to shape implants that perfectly matched the macroscopic geometry and size of bone defects, recent research ambitions include the scaffold microarchitecture for better osteoconductive properties [82].

Medical uses of $3 \mathrm{D}$ printing are becoming also common in soft-tissue applications, including cardiovascular applications, where surgical planning of complex cases and the selection of proper anatomical access is important [83]. Current $3 \mathrm{D}$ printing technologies allow the precise deposition of biocompatible materials, while at the same time potentially providing mechanical properties for optimal compliance match to the recipient surrounding tissue. Today, a wide range of $3 \mathrm{D}$ printable biomaterials is available, such as cell-laden hydrogel bioinks [84], which provide proper cell environment but still lack sufficient mechanical scaffolding properties, or biocomposite scaffold materials that can be produced through fused deposition modeling [85]. 3D printing can be used also for improving medical imaging technologies [86] and for producing anthropomorphic phantoms with realistic radiation attenuation properties [87].

Nonetheless, the wide range of possibilities of $3 \mathrm{D}$ printing do not come without limitations. While the manufacturing process is fully-automated and fast, post-processing still requires extensive manual labor. Further, the possibility to combine different $3 \mathrm{D}$ printing technologies, and, thus, to produce parts with different material properties and scales, is still in its infancy. Therefore, further evolution of the current technologies is to needed. Here, so-called "4D printing" (dynamic 3D printing) holds promise [88].

\section{IMAGING IN NON-MEDICAL APPLICATIONS}

Finally, the field of medical imaging had a profound influence also on other fields of physics and disciplines far outside those liaised with medicine, such as Anthropology, Archeology, Biology, Geophysics, Hydrology, Material Sciences and welllogging. Here, we can only mention a few developments, which turned into game changers in their field. For example, Xray and CT-imaging, as well as optical imaging, are used in paleoanthropology and archeology to image skulls, bones and whole mummies non-destructively, thus, creating new fields of "Virtual Anthropology" [89] and "Radiology in Archeology" [90]. Neutron imaging and X-ray micro-tomography, on the other hand, are used in material sciences to assess $3 \mathrm{D}$ microstructure non-destructively, as compared to optical or electronic microscope techniques that can provide only $2 \mathrm{D}$ information, and frequently in a destructive manner [91]. NMR-based well-logging, already introduced in the 1980s, caused a revolution in the petroleum industry. Parameters like permeability, mineral-independent total porosity, water, gas and oil saturation, and oil viscosity became accessible through this technology [92]. Thanks to the availability of high magnetic field strengths MRI [3] porous materials can be assessed for nonmedical purposes as well [93]. It is, therefore, interesting to note that physics discoveries from the 1900's helped to develop not only imaging techniques and, subsequently shaped the field of diagnostic imaging in medicine, but that technological advances in the diagnostic domain contributed to many useful applications outside medicine in return.

\section{OUTLOOK}

Medical imaging and therapies benefit from the close integration of physics expertise and cross-disciplinary engagement of medical physicists and biomedical engineers. The examples provided above are a testimony of the notion that medical physics is a pillar of medicine; frequently physics and medicine even leap frog each other when introducing a methodological innovation to clinical routine or when seeking to support a clinical need with a suitable and viable imaging technique, for example [27]. Some people argue for physics-driven innovation being "more risky than clinically driven development" [94]. Either way, there is an increasing awareness for medical physics symbolizing a 
"multiplicity" of disciplines at the intersection of medicine and sciences (see Figure 2 in Bailey [27]).

If accepted, this cross-domain engagement of medical physics could serve as a seed point to multidisciplinary team work as part of a suitable provision of health care for diagnostic and therapeutic purposes. Nuclear medicine physicists, for example, along with specialized radiochemists, provide the scientific basis for the use of the nuclear medicine procedures that are used in patients. They are experts in the interaction of radiation in the body and work closely with the specialist physicians and technologists in delivering treatment plans for individual patients. They are highly qualified professionals with extensive training in mathematics, radiation physics, and biological systems. They have responsibilities in the clinical environment for ensuring that all radioactive materials and equipment are accurately and safely used by the clinicians. They are often called upon to problem-solve unusual clinical presentations or results, and to develop new techniques for assessing organ function and understanding abnormal processes. They oversee equipment operation, radiation safety and protection of staff, patients and carers, teaching and training of junior scientists and medical staff, advanced IT and computing applications, troubleshooting of artifacts or abnormal appearances in scans, and verifying the results of clinical studies where measurements involving radiation are used. While medical physicists often spend a significant amount of time involved in problem-solving activities, creativity in solving the problems or devising new imaging tests and analyses is also a requirement. Being immersed in a highlyspecialized clinical environment usually requires them to become familiar with the clinical basis of many diseases and disorders so as to be able to contribute to the multidisciplinary teams managing the patients. As such, the definition of the scope of a medical physicist's profession as provided by EFOMP, falls short of the true value and training virtues.

Multi-dimensional and -disciplinary engagement will be further promoted through the recent technical and methodological innovations that have entered the market. The rapid promotion and adoption of AI mandates all partners to expand their knowledge into the area of harmonized and standardized biomarker acquisition; the promise of non-imaging biomarkers, such as those derived from liquid biopsies, mandates imaging specialists to open up to other, non-imaging based parameters. Ultimately, it is about "partnership" of medical physics and medicine, and imaging is a playground with little confrontation.

Our journal, Frontiers in Physics-Medical Physics and Imaging, seeks to provide a forum that captures the bespoke cross-disciplinary engagement of medical physicists and imaging experts. We seek to support studies that address Clinical Applications, where multi-disciplinary teams are involved in the imaging of the patients.

While the clinicians may recognize abnormal patterns due to disease, the physicist is the one expected to understand the complete diagnostic or therapeutic chain from the radioactive compound used through to the most common imaging modalities, computer analysis and interpretation of results, and to recognize any divergence from normal operations. Further, we seek to highlight relevant progress in Instrumentation, where medical physicists and engineers help ensure the correct operation of all equipment, including reference doses and measurements, accurate calibration of the imaging systems, and on to the software programs that are used to extract information from the images to provide the clinical results. Together with our partner subspecialty journal we provide a publication forum to Computing and image analysis, built on sophisticated algorithms that are used to perform functions such as three-dimensional image reconstruction, modeling of kinetic physiological systems, predicting cell kill and tissue damage from the radiation delivered to abnormalities such as cancerous deposits or to normal tissues which may also take up the therapeutic compound, combining data from separate imaging systems and multi-modality data analytics.

Next to the positive perspective on the breadth of medical (imaging) physics, we shall not abstain from providing a platform to experts discussing the challenges that come along our specialty in the next decade. In the short term, telecommuting or hybrid work models will likely be adopted in many facilities across the world to minimize the impact of COVID-19 [95]. This will require changes in the ways medical physicists perform their routine duties and how they interact with the other members of the team. As stated earlier, Artificial intelligence is increasingly being used in healthcare and has already positively affected several imaging applications (e.g., deep learning-based attenuation correction, image enhancement, machine learningenabled characterization of disease processes from multimodal datasets, etc.). It is likely that AI-enhanced hardware will also become available in the near future. While there is substantial excitement about the clinical adoption of AI-enriched next generation software and hardware, it is also true that the role of the medical physicists for many of the routine tasks performed today will need to change [96]. Physicists will not only have to stay abreast of and embrace these rapidly paced advances in AI but should also strive to take a leadership role in their clinical adoption.

The current COVID-19 situation might also change the way we interact with other disciplines: the typical annual meeting per society where live interaction is performed is replaced by online presence, which may make discussion less interactive but on the other hand opens the possibilities for researchers and experts from other disciplines to join and attend the meeting, which they would normally not travel too. Naturally, enhancing crossdiscipline communication and interaction will lead to more and transparent collaboration.

In general, the application of physics will face two significant challenges in the future. First, we need to help integrate the medical physicist into the multidisciplinary clinical team managing the twenty-first-century patient by understanding the disease, its pathological, genomic and biochemical basis, and the role that diagnostic imaging and therapeutic, or theranostic approaches can play in managing the patient. And, second, we need to expand our knowledge of the interaction of ionizing and electro-magnetic radiation with the body and the fundamental changes it induces in order to make the most effective and safe use of the powerful tools that we now have at our 
disposal. These challenges demand that the medical physicist learns to speak the language of their clinical colleagues and to be able to offer insight into the likely outcome for a patient subjected to diagnostic and therapeutic procedure. Our journal can help in this communication through the open-access publication of peer-reviews, high-quality science and opinion statements. All of us who support his endeavor would hope that this ambitious plan resonates with you as a potential reader of our journal.

\section{REFERENCES}

1. Keevil SF. Physics and medicine: a historical perspective. Lancet. (2012) 379:1517-24. doi: 10.1016/S0140-6736(11)60282-1

2. Townsend D, Cheng Z, Georg D, Drexler W, Moser E. Grand challenges in biomedical physics. Front Physics. (2013). doi: 10.3389/fphy.2013.00001

3. Moser E, Laistler E, Schmitt F, Kontaxis G. Ultra-high field NMR and MRIthe role of magnet technology to increase sensitivity and specificity. Front Phys. (2017) 5:33. doi: 10.3389/fphy.2017.00033

4. Schnerr RS, Jansen JFA, Uludag K, Hofman PAM, Wildberger JE, van Oostenbrugge RJ, et al. Pulsatility of lenticulostriate arteries assessed by 7 tesla flow MRI-measurement, reproducibility, and applicability to aging effect. Front Physiol. (2017) 8:961. doi: 10.3389/fphys.2017.00961

5. Sarracanie M, Salameh N. Low-field MRI: how low can we go? A fresh view on an old debate. Front Phys. (2020) 8:172. doi: 10.3389/fphy.2020.00172

6. Stanciu SG, Silien C, Bianchini P. Editorial: advances in label free tissue imaging with laser scanning microscopy techniques. Front Phys. (2020) 8:17. doi: 10.3389/fphy.2020.00017

7. Cai F, Gao M, Li J, Lu W, Wu C. Compact dual-channel (Hyperspectral and Video) endoscopy. Front Phys. (2020) 8:110. doi: 10.3389/fphy.2020.00110

8. Oelschlegel AM, Goldschmidt J. Functional neuroimaging in rodents using cerebral blood flow SPECT. Front Phys. (2020) 8:152. doi: 10.3389/fphy.2020.00152

9. Jones JS, Small DM, Nishimura N. In vivo calcium imaging of cardiomyocytes in the beating mouse heart with multiphoton microscopy. Front Phys. (2018) 9:128. doi: 10.3389/fphys.2018.00969

10. Moran CM, Thomson AJW. Preclinical ultrasound imaging-a review of techniques and imaging applications. Front Phys. (2020) 8:124. doi: 10.3389/fphy.2020.00124

11. Goudot G, Mirault T, Khider L, Pedreira O, Cheng C, Porée J, et al. Carotid stiffness assessment with ultrafast ultrasound imaging in case of bicuspid aortic valve. Front Physiol. (2019) 10:1330. doi: 10.3389/fphys.2019.01330

12. Schlemmer A, Berg S, Lilienkamp T, Luther S, Parlitz U. Spatiotemporal permutation entropy as a measure for complexity of cardiac arrhythmia. Front Phys. (2018) 6:39. doi: 10.3389/fphy.2018.00039

13. Karhula SS, Finnil,ä M. A., Freedman JD, Kauppinen S, Valkealahti M, Lehenkari $\mathrm{P}$, et al. Micro-scale distribution of CA4+ in ex vivo human articular cartilage detected with contrast-enhanced micro-computed tomography imaging. Front Phys. (2017) 5:38. doi: 10.3389/fphy.2017.00038

14. Schwarcz HP, Abueidda D, Jasiuk I. The ultrastructure of bone and its relevance to mechanical properties. Front Phys. (2017) 5:39. doi: 10.3389/fphy.2017.00039

15. Kurfürst A, Henits P, Morin C, Abdalrahman T, Hellmich C. Bone ultrastructure as composite of aligned mineralized collagen fibrils embedded into a porous polycrystalline matrix: confirmation by computational electrodynamics. Front Phys. (2018) 6:125. doi: 10.3389/fphy.2018.00125

16. Gagliardo C, Marrale M, D’Angelo C, Cannella R, Collura G, Iacopino G, et al. Transcranial magnetic resonance imaging-guided focused ultrasound treatment at $1.5 \mathrm{~T}$ : a retrospective study on treatment- and patientrelated parameters obtained from 52 procedures. Front Phys. (2020) 7:223. doi: $10.3389 /$ fphy.2019.00223

17. Iafrate M, Fruhwirth GO. How non-invasive in vivo cell tracking supports the development and translation of cancer immunotherapies. Front Physiol. (2020) 11:154. doi: 10.3389/fphys.2020.00154

\section{AUTHOR CONTRIBUTIONS}

All authors contributed to the content and editing and reviewing.

\section{ACKNOWLEDGMENTS}

The authors thank Carmen Boigner, Medical University Vienna for her support with this manuscript.

18. Beyer T, Bidaut L, Dickson J, Kachelriess M, Kiessling F, Leitgeb R, et al. What scans we will read: imaging instrumentation trends in clinical oncology. Cancer Imaging. (2020) 20:38. doi: 10.1186/s40644-020-00312-3

19. Kalender WA, Seissler W, Klotz E, Vock P. Spiral volumetric CT with single-breath-hold technique, continuous transport, and continuous scanner rotation. Radiology. (1990) 176:181-3. doi: 10.1148/radiology.176.1.2353088

20. Reiner CS, Roessle M, Thiesler T, et al. Computed tomography perfusion imaging of renal cell carcinoma: systematic comparison with histopathological angiogenic and prognostic markers. Invest Radiol. (2013) 48:183-91 doi: 10.1097/RLI.0b013e31827c63a3

21. Willemink MJ, Noël PB. The evolution of image reconstruction for CTfrom filtered back projection to artificial intelligence. Eur Radiol. (2019) 29:2185-95 doi: 10.1007/s00330-018-5810-7

22. Willemink MJ, Persson M, Pourmorteza A, et al. (2018) Photon-counting CT: technical principles. Radiology. doi: 10.1148/radiol.2018172656

23. Bendriem B, Townsend DW. The Theory and Practice of $3 D$ PET. Springer (1998) 168p doi: 10.1007/978-94-017-3475-2

24. Gundacker S, Heering A. The silicon photomultiplier: fundamentals and applications of a modern solid-state photon detector. Phys Med Biol. (2019). doi: 10.1088/1361-6560/ab7b2d

25. Spencer BA, Berg E, Schmall JP, Omidvari N, Leung EK, Abdelhafez YG, et al. Performance evaluation of the Uexplorer total-body PET/CT scanner based on NEMA NU 2-2018 with additional tests to characterize long axial field-of-view PET scanners. J Nucklear Medizin. (2020). doi: 10.2967/jnumed.120.250597

26. Sakai M, Kubota Y, Kumar R, Kikuchi M, Arakawa K, Nakano T. (2019) Compton imaging with $99 \mathrm{~m}$ Tc for human imaging. Sci Rep. doi: 10.1038/s41598-019-49130-z

27. Bailey DL. Thirty years from now: future physics contributions in nuclear medicine. EJNMMI Phys. (2014). doi: 10.1186/2197-7364-1-4

28. Shung KK. High frequency ultrasonic imaging. Med Ultrasound J. (2009) 17:25-30. doi: 10.1016/S0929-6441(09)60012-6

29. Qin S, Caskey CF, Ferrara KW. Ultrasound contrast microbubbles in imaging and therapy: physical principles and engineering. Phys Med Biol. (2009) 54:R27-57. doi: 10.1088/0031-9155/54/6/R01

30. Qu Y, Meng Y, Feng S, Liu M, Xiao L, Zhang X, et al. Therapeutic assessment of high-intensity focused ultrasound for vulvar lichen sclerosus by active dynamic thermal imaging and hyperspectral imaging-a preliminary study. Front Phys. (2020) 8:91. doi: 10.3389/fphy.2020.00091

31. Mériaux S, Conti A, Larrat B. Assessing diffusion in the extra-cellular space of brain tissue by dynamic MRI mapping of contrast agent concentrations. Front Phys. (2018) 6:38. doi: 10.3389/fphy.2018.00038

32. Wang $\mathrm{C}, \mathrm{Li} \mathrm{X}, \mathrm{Hu} \mathrm{H}$, Zhang L, Huang Z, Lin M, et al. Monitoring of the central blood pressure waveform via a conformal ultrasonic device. Nat BME. (2018) 2:687-95. doi: 10.1038/s41551-018-0 287-x

33. Ashhar K, Soh CB, Kong KH. A wearable ultrasonic sensor network for analysis of bilateral gait symmetry. In: 2017 39th Annual International Conference of the IEEE Engineering in Medicine and Biology Society (EMBC). Jeju: IEEE (2017). p. 4455-8. doi: 10.1109/EMBC.2017.803 7845

34. Liu S, Wang Y, Yang X, Lei B, Liu L, Xiang Li S, et al. Deep learning in medical ultrasound analysis: a review. Engineering. (2018) 5:26175. doi: 10.1016/j.eng.2018.11.020 
35. Lauterbur PC. Image formation by induced local interactions: examples employing nuclear magnetic resonance. Nature. (1973) 242:190-1. doi: 10.1038/242190a0

36. Niesporek SC, Nagel AM, Platt T. Multinuclear MRI at ultrahigh fields. Top Magn Reson Imaging. (2019) 28:17388. doi: 10.1097/RMR.0000000000000201

37. Van der Velden T, Rivera D, Hendrikse A, Siero J, Klomp D. Inventors method and apparatus for ultrasonic gradients in magnetic resonance imaging. European Patent Application EP 3. (2018) 364 205A1

38. Alsop DC, Detre JA, Golay X, Gunther M, Hendrikse J, Hernandez-Garcia L, et al. Recommended implementation of arterial spin-labeled perfusion MRI for clinical applications: a consensus of the ISMRM perfusion study group and the European consortium for ASL in dementia. Magn Reson Med. (2015) 73:102-16. doi: 10.1002/mrm.25197

39. Rowley HA, Grant PE, Roberts TP. Diffusion MR imaging. Theory and applications. Neuroimaging Clin N Am. (1999). 9:343-61

40. Hangel G, Strasser B, Povazan M, Heckova E, Hingerl L, Boubela $\mathrm{R}$, et al. Ultra-high-resolution brain metabolite mapping at $7 \mathrm{~T}$ by short-TR Hadamard-encoded FID-MRS. Neuroimage. (2018) 168:199210. doi: 10.1016/j.neuroimage.2016.10.043

41. Vladymyrov M, Haghayegh Jahromi N, Kaba E, Engelhardt B, Ariga A. VivoFollow 2: distortion-free multiphoton intravital imaging. Front Phys. (2020) 7:222. doi: 10.3389/fphy.2019.00222

42. Martínez-Ojeda RM, Pérez-Cárceles MD, Ardelean LC, Stanciu SG, Bueno JM. Multiphoton microscopy of oral tissues: review. Front Phys. (2020) 8. doi: 10.3389/fphy.2020.00128

43. Karunamuni GH, Gu S, Ford MR, Peterson LM, Ma P, Wang YT, et al. Capturing structure and function in an embryoic heart with biophotonic tools. Front Phys. (2014) 5:351. doi: 10.3389/fphys.2014.00351

44. Jendzjowsky NG, Steinback CD, Herman RJ, Tsai WH, Costello FE, Wilson RJA. Functional-optical coherence tomography: a non-invasive approach to assess the sympathetic nervous system and intrinsic vascular regulation. Front Phys. (2019) 10:1146. doi: 10.3389/fphys.2019.01146

45. Imamura $\mathrm{T}$, Saitou $\mathrm{T}$, Kawakami $\mathrm{R}$. In vivo optical imaging of cancer cell function and tumor microenvironment. Cancer Sci. (2018) 109:9128. doi: $10.1111 /$ cas. 13544

46. Li X, Qiao $\mathrm{H}, \mathrm{Wu} J$, Lu Z, Yan $\mathrm{T}$, Zhang $\mathrm{R}$, et al. DeepLFM: deep learning-based $3 \mathrm{D}$ reconstruction for light field microscopy in novel techniques in microscopy (Optical Society of America), NM3C-2. (2019). doi: 10.1364/NTM.2019.NM3C.2

47. Wheelock MD, Culver JP, Eggebrecht AT. High-density diffuse optical tomography for imaging human brain function. Rev Sci Instrum. (2019) 90:051101. doi: 10.1063/1.5086809

48. Rieckher M, Birk UJ, Meyer H, Ripoll J, Tavernarakis N. Microscopic optical projection tomography in vivo. PLoS ONE. (2011) 6:e18963. doi: 10.1371/journal.pone.0018963

49. Nehrhoff I, Ripoll J, Samaniego R, Desco M, Gómez-Gaviro MV. Looking inside the heart: a see-through view of the vascular tree. Biomed Opt Express BOE. (2017) 8:3110-8. doi: 10.1364/BOE.8.003110

50. Leitgeb RA, Baumann B. Multimodal optical medical imaging concepts based on optical coherence tomography. Front Phys. (2018) 6. doi: 10.3389/fphy.2018.00114

51. Cremer C, Birk, U. Perspectives in super-resolved fluorescence microscopy: what comes next? Front Phys. (2016) 4:11. doi: 10.3389/fphy.2016.00011

52. Liu X, Tu S, Xu Y, Song H, Liu W, Liu Q, et al. Aberrations in structured illumination microscopy: a theoretical analysis. Front Phys. (2020) 7:254. doi: 10.3389/fphy.2019.00254

53. Hu Z, Chen WH, Tian J. Cheng Z. NIRF nanoprobes for cancer molecular imaging: approaching clinic. Trends Mol Med. (2020) 26:46982. doi: 10.1016/j.molmed.2020.02.003

54. Hu Z, Fang C, Li B, Zhang Z, Cao C, Cai M, et al. First-in-human liver-tumour surgery guided by multispectral fluorescence imaging in the visible and near-infrared-I/II windows. Nat Biomed Eng. (2020) 4:25971. doi: 10.1038/s41551-019-0494-0

55. Suo Y, Wu F, Xu P, Shi H, Wang T, Liu H. Cheng Z. NIR-II fluorescence endoscopy for targeted imaging of colorectal cancer. Adv Healthc Mater. (2019) 8:e1900974. doi: 10.1002/adhm.201900974
56. van Sluis J, de Jong J, Schaar J, Noordzij W, van Snick P, Dierckx R, et al. Performance characteristics of the digital biograph vision PET/CT System. $J$ Nucl Med. (2019) 60:1031-6. doi: 10.2967/jnumed.118.215418

57. Nguyen NC, Vercher-Conejero JL, Sattar A, Miller MA, Maniawski $\mathrm{PJ}$, Jordan DW, et al. Image qualitiy and diagnostic performance of a digital PET prototype in patients with oncologic diseases: intitial experience and comparison with analog PET. J Nucl Med. (2015) 56:137885. doi: 10.2967/jnumed.114.148338

58. Cherry SR, Jones T, Karp JS, Qi J, Moses WW, Badawi RD. Total-body PET: maximizing sensitivity to create new opportunities for clinical research and patient care. J Nucl Med. (2018) 59:3-12. doi: 10.2967/jnumed.116.184028

59. Badawi RD, Shi H, Hu P, Chen S, Xu T, Price PM, et al. First human imaging studies with the EXPLORER total-body PET scanner. J Nucl Med(2019) 60:299-303. doi: 10.2967/jnumed.119.226498

60. Ljungberg $M$, Pretorius $H$. SPECT/CT: an update on technological developments and clinical applications. Br J Radiol. (2016) 91:20160402. doi: 10.1259/bjr.20160402

61. Valladares A, Ahangari S, Beyer T, Boellaard R, Chalampalakis Z, Comtat $\mathrm{C}$, et al. Clinically valuable quality control for PET/MRI systems: consensus recommendation from the HYBRID Consortium. Front Phys. (2019). doi: 10.3389/fphy.2019.00136

62. Hacker M, Hicks RJ, Beyer T. Applied Systems biology-embracing molecular imaging for systemic medicine. EJNMMI. (2020) 47:27215. doi: 10.1007/s00259-020-04798-8

63. Osborne DR, Kuntner C, Berr S. Guidance for efficient small animal imaging quality control. Mol Imaging Biol. (2017) 19:485-98. doi: 10.1007/s11307-016-1012-3

64. Walter A, Paul-Gilloteaux P, Plochberger B, Sefc L, Verkade P, Mannheim JG, et al. Correlated multimodal imaging in life sciences: expanding the biomedical horizon. Front Phys. (2020) 8:47. doi: 10.3389/fphy.2020.00047

65. Grau C, Durante M, Georg D, Langendijk JA, Weber DC. Particle therapy in Europe. Mol Oncol. (2020) 14:1492-9 doi: 10.1002/1878-0261.12677

66. Gregoire V, Guckenberger M Haustermans K, Lagendijk JJW, Ménard C, Pöter R, et al. Image guidance in radiation therapy for better cure of cancer. Mol Oncol. (2020) 14:1470-91. doi: 10.1002/1878-0261.12751

67. Bertholet J, Knopf A, Eiben B, McClelland J, Grimwood A, Harris E, et al. Realtime intrafraction motion monitoring in external beam radiotherapy. Phys Med Biol. (2019) 64:15TR01. doi: 10.1088/1361-6560/ab2ba8

68. Hoisak JDP, Pawlicki T. The role of optical surface imaging systems in radiation therapy. Semin Radiat Oncol. (2018) 28:185-93. doi: 10.1016/j.semradonc.2018.02.003

69. Chin S, Eccles CL, McWilliam A, Chuter R, Walker E, Whitehurst P, et al. Magnetic resonance guided radiation therapy: a review. J Med Imaging Radiat Oncol. (2020) 64:163-77. doi: 10.1111/1754-9485.12968

70. Hall WA, Paulson ES, van der Heide U, Fuller CD, Raaymakers BW, Lagendijk JJW, et al. The transformation of radiation oncology using realtime magnetic resonance guidance: a review. Eur J Cancer. (2019) 122:4252. doi: 10.1016/j.ejca.2019.07.021

71. Lustberg T, van Soest J, Gooding M, Peressutti D, Aljabar P, van der Stoep J, et al. Clinical evaluation of atlas and deep learning bases automatic contouring for lung cancer. Radiother Oncol. (2018) 126:3127. doi: 10.1016/j.radonc.2017.11.012

72. Gooding MJ, Smith AJ, Tariq M, Aljabar P, Peressutti D, van der Stoep $\mathrm{J}$, et al. Comparative evaluation of autocontouring in clinical practice: A practical method using the Turing test. MedPhys. (2018) 45:510515. doi: $10.1002 / \mathrm{mp} .13200$

73. Terpstra ML, Maspero M, d'Agata F, Stemkens B, Intven MPW, Lagendijk JJW, et al. Deep learning-based image reconstruction and motion estimation from undersampled radial k-space for real-time MRI-guided radiotherapy. Phys Med Biol. (2020) 65:155015. doi: 10.1088/1361-6560/ab9358

74. Yuan N, Dyer B, Rao S, Chen Q, Bededict S, Shang L, et al. Convolutional neural network enhancement of fast-scan low-dose conebeam CT images for head and neck radiotherapy. Phys Med Biol. (2020) 65:035003. doi: 10.1088/1361-6560/ab6240

75. Fan J, Wang J, Chen Z, Hu C, Zhang Z, Hu W. Automatic treatment planning based on three-dimensional dose distribution predicted from deep learning technique. Med Phys. (2018) 46:370-81. doi: 10.1002/mp.13271 
76. Fiorino C, Muren LP, Clark CH, van Elmpt W, Jornet N. Expanding the scientific role of medical physics in radiotherapy: time to act. Radiother Oncol. (2015) 117:401-2. doi: 10.1016/j.radonc.2015.11.007

77. Bortfeld T, Jeraj R. The physical basis an dfuture of radiation therapy. $\mathrm{Br} J$ Radiol. (2011) 84: 485-98. doi: 10.1259/bjr/86221320

78. Fiorino C, Jeraj R, Clark CH, Garibaldi C, Georg D, Muren L, et al. Grand challenges for medical physics in radiation oncology. Radiotherapy Oncol. (2020) 8:7-17. doi: 10.1016/j.radonc.2020.10.001

79. Vandewinckele L, Claessens M, Dinkla A, Brouwer C, Crijns W, Verellen D, et al. Overview of artificial intelligence-based applications in radiotherapy: Recommendations for implementation and quality assurance. Radiotherapy Oncol. (2020) 153:55-66. doi: 10.1016/j.radonc.2020.09.008

80. Vidal L, Kampleitner C, Brennan MA, Hoornaert A, Layrolle P. Reconstruction of large skeletal defects: current clinical therapeutic strategies and future directions using 3D printing. Front Bioeng Biotechnol. (2020) 8:61. doi: $10.3389 /$ fbioe. 2020.00061

81. Oberoi G, Nitsch S, Edelmayer M, Janjic K, Müller AS, Agis H. 3D Printing - Encompassing the facets of dentistry. Front Bioeng Biotechnol. (2018) 6:172. doi: 10.3389/fbioe.2018.00172

82. Ghayor C, Weber FE. Osteoconductive microarchitecture of bone substitutes for bone regeneration revisited. Front Phys. (2018) 9:960. doi: 10.3389/fphys.2018.00960

83. Batteaux C, Haidar MA, Bonnet D. 3D-printed models for surgical planning in complex congenital heart diseases: a systematic review. Front Pediatrics. (2019) 7:23. doi: 10.3389/fped.2019.00023

84. Ramiah P, du Toit LC, Choonara YE, Kondiah PPD, Pillay V. Hydrogelbased bioinks for 3D bioprinting in tissue regeneration. Front Mater. (2020) 7:76. doi: $10.3389 /$ fmats. 2020.00076

85. Wasti S, Adhikari S. Use of biomaterials for $3 \mathrm{D}$ printing by fused deposition modeling technique: a review. Front Chem. (2020) 8:315. doi: $10.3389 /$ fchem.2020.00315

86. Gerges T, Semet V, Lombard P, Gaillard S, Cabrera M, Lambert SA. 3D Plastronics for smartly integrated magnetic resonance imaging coils. Front Phys. (2020) 8:240. doi: 10.3389/fphy.2020.00240

87. Hatamikia S, Oberoi G, Unger E, Kronreif G, Kettenbach J, Buschmann $\mathrm{M}$, et al. Additively manufactured patient-specific anthropomorphic thorax phantom with realistic radiation attenuation properties. Front Bioeng Biotechnol. (2020) 8:385. doi: 10.3389/fbioe.2020.00385
88. Tamay DG, Usal TD, Alagoz AS, Yucel D, Hasirci N, Hasirci V. 3D and $4 \mathrm{D}$ printing of polymers for tissue engineering applications. Front Bioeng Biotechnol. (2019) 7:164. doi: 10.3389/fbioe.2019.00164

89. Uldin T. Virtual anthropology - a brief review of the literature and history of computed tomography. Forensic Sci Res. (2017) 2:165-73. doi: 10.1080/20961790.2017.1369621

90. Licata M, Pinto A. Radiology in Archaeology: Fundamentals PerspectiveExamination of the Living. In: Lo Re G., Argo A., Midiri M., Cattaneo C. (eds) Radiology in Forensic Medicine. Cham: Springer (2020).

91. Salvo L, Suéry M, Marmottant A, Limodin N, Bernard D. 3D imaging in material science: application of X-ray tomography. Comptes Rendus Physique. (2010) 11:641-9. doi: 10.1016/j.crhy.2010.12.003

92. Coates GR, Xiao L, Prammer MG. NMR Logging Principles and Applications. Ann Arbor, MI: University of Michigan; Elsevier Science (1999).

93. Blunt MJ. Multiphase Flow in Permeable Media. Cambridge: Cambridge University Press (2017). doi: 10.1017/9781316145098

94. Watson CC. The dynamics of physics in PET. EJNMMI Physics. (2014). doi: 10.1186/2197-7364-1-6

95. Lincoln H, Khan R, Cai J. Telecommuting: A viable option for medical physicists amid the COVID-19 outbreak and beyond. Med Phys. (2020) 74:2045-8. doi: 10.1002/mp.14203

96. Kortesniemi N, Tsapaki V, Trianni A, Russo P, Maas A, Källman HE, et al. White paper: big data and deep learning in medical imaging and in relation to medical physics profession. EJNMP. (2018) 56:903. doi: 10.1016/j.ejmp.2018.11.005

Conflict of Interest: The authors declare that the research was conducted in the absence of any commercial or financial relationships that could be construed as a potential conflict of interest.

Copyright (c) 2021 Beyer, Bailey, Birk, Buvat, Catana, Cheng, Fang, Giove, Kuntner, Laistler, Moscato, Nekolla, Rausch, Ronen, Saarakkala, Thielemans, van Elmpt and Moser. This is an open-access article distributed under the terms of the Creative Commons Attribution License (CC BY). The use, distribution or reproduction in other forums is permitted, provided the original author(s) and the copyright owner(s) are credited and that the original publication in this journal is cited, in accordance with accepted academic practice. No use, distribution or reproduction is permitted which does not comply with these terms. 